\title{
LA LEY GENERAL DE EDUCACIÓN (LGE) DE 1970 / CINCUENTA AÑOS DE RECUERDOS...
}

\author{
The General Education Act of 1970 / Fifty Years \\ of memories....
}

\section{Marc Antoni Adell Cueva ${ }^{\beta}$}

Hecha la principal salvedad, en la primera nota a pie de página, parece oportuno remitir a los lectores, al interesante estudio del profesor Mayordomo que defiende que «las huellas o muestras que conservamos de nuestro pasado educativo» pueden llegarnos a través de «la palabra viva de actores y/o testigos del mismo", en forma de "historias de vida o relatos biográficos», que «sin duda presentan problemas y limitaciones, pero también ofrecen oportunidades para abundar en esa constante búsqueda de integración entre historia, objetividad y verdad». ${ }^{1}$ Aunque sobre ello volveremos más adelante.

Está(ba)mos en mayo del 68, en pleno protagonismo de la «movida» estudiantil de París -que despertó más expectativas de las que logró alcanzar-, mientras otros estudiantes -los de quinto año, de la $1^{\mathrm{a}}$ promoción de Pedagogía de València- atisbábamos el final de curso -y carrera-,

\footnotetext{
${ }^{a}$ La presente colaboración no es sino una serie de vivencias, experimentadas por un joven -entonces- inspector de educación, recién accedido a la función inspectora, a cuyos integrantes se les «comisionó» -más o menos formalmente- en su difusión y aplicación -que no en su evaluación, atribuida a los ICEs- de la LGE, y que vivió aquella experiencia intensamente, bien que con toda la subjetividad atribuible. Así pues, un «relato» objetivo, documentado y de rigor histórico de la LGE -en sus 50 años: 1970-2020- habrá que buscarlo en otra parte: el estudio del profesor Manuel de Puelles Benítez, recogido en la obra Política y Educación en la España Contemporánea (Madrid: UNED, 2004), me parece clarividente. Obviamente, otros artículos que integran el presente monográfico son, también y del todo recomendables, al respecto.

B Inspector de Educación. Profesor Sènior de la Universitat de València. Dpto. de Psicología Social. Avda. Blasco Ibàñez, 21, 4ª planta. 46010 Valéncia. España. marc.adell@uv.es

1 Alejandro Mayordomo, «El legado de las voces y los escritos: su valor como testimonio de la cultura escolar». Revista Iberoamericana do Patrimônio Educativo 6 (2020): 3.
} 
con el consiguiente sobreesfuerzo de pruebas y entrega de trabajos para obtener el título y (re)plantearnos el futuro. ${ }^{2}$ Así fue cómo algunos nos matriculábamos en las recién convocadas oposiciones de Inspección que incluyeron aquel año, un nuevo ejercicio -eliminatorio, como todos- de lengua inglesa, añadida al francés de siempre. ${ }^{3}$

Sea como fuere algunos teníamos idealizada ${ }^{4}$ la figura del inspector -mayoritariamente inspectora- y el encuentro casual con mi vecino y antiguo profesor de Matemáticas de la Normal de Castelló, Dn. Felipe Sáiz, a quien comuniqué mi propósito de presentarme a las pruebas, lo corroboró. ${ }^{5}$ En otra visita obligada -al director de la Escuela y profesor

\footnotetext{
$2 \mathrm{Al}$ respecto el profesor Benavent, que nos impartía Orientación Escolar y Vocacional, ya nos había testado a cada uno, respecto a qué queríamos ser de «mayor», que en mi caso contesté en el correspondiente espacio de la respuesta a su pregunta con «cátedra de investigación en Escuela Normal» (sic). Lamentablemente, con el anuncio de la futura Ley General de Educación -coloquialmente «ley Villar»- y la subsiguiente aparición del «Libro Blanco», que preveía serios cambios en el sistema -también universitario-, aquellas plazas, a las que yo aspiraba, quedaron «congeladas». Sí, en cambio, se hablaba de la última convocatoria, según el formato de entonces, de oposiciones a Inspección.

3 Y que nos supuso un esfuerzo suplementario, pues los de aquellas promociones, no habíamos cursado otro idioma «moderno» que el francés.

4 Será casualidad, o no, pero algunos no tuvimos ninguna experiencia desagradable, en el trato con los inspectores que me giraron visita, más bien interesante y fluida a la par que útil, para mejorar mi dedicación a la clase. Así, de resultas, me sentía agradecido y mantuve con todos ellos una relación de amistad y confianza, no exenta de respeto al «superior», claro.
}

5 El bueno de Dn. Felipe exclamó: «la Inspección, jel generalato del magisterio!», con lo que reforzaba mi aspiración de forma contundente y hasta admirativa, desde su condición de profesor numerario de Escuela Normal, mi primera pretensión vocacional, Hay que decir que durante mis estudios de Pedagogía, había recurrido a él en demanda de sugerencias, materiales y experiencias, en tanto que antiguo alumno de la Escuela Superior de Magisterio de Madrid. Vid: https://es.wikipedia.org/ wiki/Escuela_de_Estudios_Superiores_de_Magisterio, centro emblemático al que accedían y en el que se formaban los futuros inspectores de Enseñanza y profesores de Normal y en la que, de entre los ilustres personalidades de la época que impartieron docencia, el profesor Sáiz recuerda a Menéndez Pidal.

Lamentablemente el centro desapareció en 1932, con ocasión de crearse la Facultad de Pedagogía de la Universidad de Madrid, mientras en Saint Cloud -modelo de la de Madrid- sigue en funcionamiento aquella École Normale Supérieure, dedicada no solo a la excelencia en la preparación de docentes, sino a la «formation par la recherche des cadres supérieurs des administrations publiques et des entreprises françaises et européennes». Vid: https://www.ens.psl.eu/l-ecole-normale-superieure/l-ens-aujourd-hui. No todo el mundo conoce el paso de algún presidente de la República Francesa -Pompidoupor aquel centro: hijo de maestros, Georges Jean Raymond Pompidou, «su vocación lo lleva a la Escuela Normal Superior, y poco después, iniciaría la carrera docente» Vid.: https://es.wikipedia.org/ wiki/Georges_Pompidou.

Por cierto, en la portada de los libros del prestigioso autor de la época y referente obligado en el ámbito de la educación del momento, Gaston Mialaret, aparecía su condición de Professeur de l'École Normale Supérieure de Saint Cloud. Como así en la referencia on line: http://www.ibe.unesco.org/es/ ibe-staff/gaston-mialaret, donde se destacan sus titulaciones académicas: «degrees in mathematics, psychology, and psycho-pedagogy", así como haber obtenido -lo que me complace especialmente- el 
de Lengua, Dn. Isidoro Andrés- me despidió con unas palabras, a las que debo, en buena medida, el acierto y satisfacción -permítaseme la inmodestia-, en el ejercicio de la función inspectora. ${ }^{6}$ Así que nos pusimos de inmediato a preparar el largo y denso temario de la oposición. ${ }^{7}$

El caso es que el tardofranquismo parecía tocar a su fin, pero aún iba dando los últimos «coletazos», unos con la contundencia represiva de siempre y otros de pretendida y tímida «democratización»: la Ley General de Educación ${ }^{8}$ (en lo sucesivo LGE) fue, en buena medida, un intento de lo segundo... O así la percibimos algunos.

Ciertamente el profesor Villar Palasí ${ }^{-}$-valenciano de Paterna, por cierto-, persona discreta y prudente, según entendía yo, no se significó -declaradamente al menos- ni como adicto al régimen, ni menos aún como "contestatario», claro. Pero, bien pudiera atribuírsele, como titular de Educación, la iniciativa del proyecto y alguna influencia en la «línea» de pensamiento de la LGE y no solo para que aquel proyecto legal pudiera parecer "progresista» ${ }^{10}$ sino para preludiar incluso una cierta

Certificate of Aptitude for the Inspection of Primary Schools, así como la condición de «the higher teacher training school in Saint-Cloud» e «in the University of La Sorbonne», para acabar "after his retirement in 1984, he was called by UNESCO to become the Director of the International Bureau of Education in Geneva».

6 «Sé de los maestros, Marc, amigo y consejero» Nada más, ni nada menos.

7 Efectivamente, cuatro colegas -uno de ellos profesor interino de Pedagogía en la Normal de Castelló-, un buen amigo maestro prestigiado y dos compañeras más, nos distribuimos la preparación de los temas, que concluyó en una más que voluminosa carpeta.

8 El título formal -inicial- era más largo y pretencioso -Ley General... y de Financiamiento de la Reforma Educativa-, pero aquel epílogo, auténtica vertebración y garantía de la propuesta, pronto «cayó» del redactado y nada más se supo. La «razón» (?) fue que no se podía condicionar el presupuesto del Estado $(i)$ a una asignación específica y gradual para garantizar la reforma educativa, cuando había tantos otros temas (i) que atender... En consecuencia, en cada ejercicio económico, se estudiaría con qué disponibilidad presupuestaria se podía contar, lo que desvirtuó aquella inicial previsión de «acompañar» la aplicación de la Ley, con su correspondiente gasto, garantizado.

9 Experto en Derecho, fue miembro de la Real Academia de Jurisprudencia y Legislación y presidente del Consejo Superior de Investigaciones Científicas (CSIC). Vid: https://es.wikipedia.org/wiki/ Jos\%C3\%A9_Luis_Villar_Palas\%C3\%AD

10 Un estudio pormenorizado del contenido de la LGE -que no es el caso-, muestra el «esfuerzo» en presentarla como una apuesta novedosa (metodología, curricula, organización, participación social en el gobierno de los centros...), aunque con el peligro detectado por de Puelles (vid. nota a), de una cierta inspiración "tecnocrática», detectada en referencias como "preparación especializada» «afrontar con eficacia las nuevas situaciones» «asesoramiento de los sectores profesionales más capacitados» «incorporación fecunda del individuo a la vida del trabajo». Y todo ello solo en la «Justificación» o Prólogo de la Ley, con la evidencia de aparecer -aquella apuesta novedosa- contaminada 
normalización democrática del régimen, al aparecer en el texto expresiones como "la preparación para el ejercicio responsable de la libertad",11 atribuida a una educación como la que se proponía en la propia Ley, así como muchas otras expresiones del mismo o parecido tenor. ${ }^{12}$ Finalmente el texto después de su «discusión» en las Cortes franquistas, vio la luz en el BOE. ${ }^{13}$ Así las cosas, la Inspección Educativa recibió el «encargo» (vid. nota núm. 1) de divulgar la LGE y «acompañar» a los protagonistas -alumnado, profesorado, expertos, autoridades y familias...- en su puesta en marcha, ${ }^{14}$ lo que, supuestamente, los inspectores jóvenes -algunos recién ingresados- asumimos con más entusiasmo que los de más edad y experiencia. ${ }^{15}$ Sea como fuere, por lo que a mí respecta y al reducido grupo de componentes de la Inspección Educativa de Girona -en una plantilla de ocho efectivos éramos cuatro- ${ }^{16}$ nos pusimos a ello y eso a través de las siguientes iniciativas: las visitas ordinarias a las escuelas, los encuentros en los recién instaurados Centros de Colaboración Pedagógica, históricamente denominados en Catalunya Cercles

\footnotetext{
por la tecnocracia económico-administrativa del momento. Veamos, algunos ejemplos más de intento de democratización y eso ya en los párrafos introductorios: "contribuir a la edificación de una sociedad más justa» con una "reforma integral de nuestro sistema educativo» contando con la "consulta a la sociedad española» y con «ánimo abierto al ensayo, a la reforma y a la colaboración» para «ofrecer a todos la igualdad de oportunidades» así como la «dignificación social y económica de la profesión docente» introduciendo "nuevos métodos y técnicas de enseñanza», etc. etc.

11 Vid.: Art. 1. ${ }^{\circ}$, Título preliminar: fines de la educación (BOE núm. 187, de 6 de agosto de 1970).

12 Incorporando referencias como «sociedad moderna», «mundo contemporáneo» «democratizar la enseñanza» -solo o porque de la política ¿valía más no hablar?- (vid.: LGE-Prólogo).

13 BOE núm. 187, de 6 de agosto de 1970, 12525 a 12546, como hemos visto.

14 Que a mi juicio no rentabilizamos bastante, a favor de recuperar el «buen nombre» de nuestro colectivo, percibido por los docentes, y buena parte de la sociedad, como de (casi) exclusiva vigilancia del cumplimiento de normas y del buen funcionamiento administrativo de los centros -inspectores «legalistas», vaya, en lugar de supervisores y favorecedores de la mejora de la calidad de la educación impartida.
}

15 Alguno de los cuales como el que «ejercía» sobre mi persona -la figura no existía formalmente- de tutor, en los primeros tiempos de mi incorporación al primer destino: la Inspección Provincial de Girona, Dn. Lluís M. ${ }^{a}$ Mestras, ilustre inspector ya en la República y exalumno de la Escuela Superior del Magisterio de Madrid -persona, por tanto, muy preparada, que se brindó a «tutelarme»y que yo aún agradezco ahora. Que, con tacto, pero asertivamente, me hizo ver que las propuestas y metodologías que algunos promovíamos, en base a la LGE, como «novedosas», no eran tan diferentes de los principios y prácticas de la Escuela Nueva y sus promotores: Claparède, Decroly, Montessori... Fue una lección de humildad que no he olvidado. Las otras dos compañeras eran María Serra, que había cursado estudios en Barcelona y Anna M. ${ }^{a}$ Oriol, en Madrid.

16 El citado Sr. Mestras, en funciones de Secretario de la plantilla, las citadas Anna M. ${ }^{a}$ Oriol, en la Jefatura y la compañera María Serra. 
d'estudi, y nuestra participación en los programas de formación y reciclaje del profesorado de educación primaria, de la Universidad Nacional de Educación a Distancia (UNED). ${ }^{17}$

Respecto a las primeras, mis colegas -de más edad y experienciatenían en los centros una reconocida autoridad, de la que carecía, por el momento el "nuevo», 18 bien que no era asunto que me preocupara, sino más bien cómo introducirme discretamente en el colectivo de docentes, despertar su curiosidad por la «reforma» y animar, y acompañar, en el decurso de su aplicación, buscando la complicidad de los directores, pieza clave en aquella pretensión. Al respecto, una iniciativa que me ha ocupado a lo largo de mi vida profesional, ha sido la de mi presencia -sugerida, pero irrenunciable en el contexto de mis «visitas»en las clases, compartiendo, breve y discretamente, la actividad del momento, con el profesor o profesora -especialmente con los tutores-, ${ }^{19}$ con la intención de reforzar su papel y autoridad, particularmente frente a los grupos más «movidos» de la clase, a los que me dirigía veladamente para advertirles que les «pediría cuentas», en mi próxima visita, de su dedicación y rendimiento. ${ }^{20}$ Obviamente, la conversación -más distendida- con el profesorado y la dirección, tras la visita, aparecía como obligada al final de la jornada y al entorno de la nueva Ley y la oportunidad que se nos brindaba de seguir mejorando la enseñanza impartida. También el trato con las familias y autoridades locales iba en la misma dirección. ${ }^{21}$

\footnotetext{
17 Iniciativa -la UNED-, al parecer del propio Dn. José Luís Villar que la promovió.

18 Con frecuencia confundido en reuniones y encuentros como «maestro», del que se inquiría la procedencia y lugar de trabajo, por parte de los asistentes.

19 La función tutorial ha estado, desde entonces, presente en mis preocupaciones y la he defendido como imprescindible para conseguir una educación integral y de calidad, especialmente en educación secundaria que es, lamentablemente, donde menos asumida está, aún ahora; de forma que algunos la calificamos a día de hoy como «la asignatura pendiente del sistema».
}

20 Aquellas experiencias, y las que siguieron, me animaron a poner por escrito algunas alternativas y propuestas de intervención, que vieron la luz en forma de libros o artículos y que creo -modestamenteque no han perdido actualidad. Vid.: http://noublocdemarc.blogspot.com/p/blog-page_21.html.

${ }_{21}$ Respecto a las primeras, las sesiones con padres y madres no siempre resultaban fluidas, pues fuera de intentar explicarles las «virtudes» de la LGE, en la práctica podía derivarse la supresión, o disminución, de las unidades escolares de la localidad, para trasladar al escaso alumnado al centro comarcal correspondiente, que no siempre era bien visto por las familias. Con las autoridades locales pasaba otro tanto, salvo si era para mejorar, ampliar o construir nuevos edificios escolares en su población. 
Respecto a los Centros de Colaboración Pedagógica procuré -procuramos- revitalizar aquellos Cercles d'estudi, con la presencia participada y colaborativa del profesorado de cada zona o comarca, con propuestas sugerentes de los ponentes invitados y aportaciones prácticas de los asistentes. ${ }^{22}$

\section{Y por lo que hace al reciclaje del profesorado a través del Pro-} grama de la UNED, nos dio la oportunidad de trabajar, codo con codo, con el profesorado de la Normal, relación del todo conveniente, más allá de compartir vocalías -o presidencia- en tribunales de oposición de magisterio. El caso es que hubimos de mantener los inspectores, como el resto de participantes en la aplicación de la LGE, un esfuerzo intelectual suplementario, en lecturas y búsqueda de fuentes, autores ${ }^{23}$ y materiales. La terminología al uso hubo que incorporarla a la conversación ordinaria y había que andarse con tiento, para no «desmerecer» frente a los interlocutores. ${ }^{24}$

Fuera de eso el ministerio inició un ambicioso programa de construcciones escolares, especialmente en las poblaciones importantes, para dar cobijo a los numerosos grupos de alumnado procedente de las pequeñas y dispersas localidades, a través de los servicios de transporte y comedor. ${ }^{25} \mathrm{Al}$ respecto del nuevo diseño de edificios escolares se

22 Las sesiones de trabajo se completaban con convivencias y visitas, para reforzar el sentido de pertenencia al colectivo y de autoestima, con presencia, a veces, de las autoridades locales para impulsar su implicación y complicidad en la aplicación de la LGE.

23 El octogenario -entonces- Piaget, cobró nuevo protagonismo, en sus trabajos de psicopedagogía y hasta recibió la visita de fin de curso, en Ginebra, de un grupo de universitarios gerundenses, estudiantes de Psicología. Y nombres como Ausubel y Novack se prodigaban, en sus teorías sobre el aprendizaje significativo. Entre nosotros cobró renombre el profesor Coll, de Barcelona, que encabezó varios grupos de trabajo y fue requerido por el Ministerio de Educación y Ciencia (MEC), como asesor del equipo ministerial del momento.

24 «Programación»: larga, a medio plazo o inmediata de clase; «curricula»; «objetivos»: finales, específicos, operativos,...; «procedimientos»: intervención, propuestas, investigación...; «contenidos»: conocimientos, actitudes, estrategias...; «actividades»: genéricas, atendida la diversidad, individuales, grupales...; “"evaluación», «recuperación», «enlaces», «preconceptos»... Y un larguísimo etcétera.

$25 \mathrm{Al}$ respecto aun mantengo una cierta mala conciencia en haber contribuido a «forzar» la aplicación de la política ministerial del momento, de favorecer la creación de concentraciones escolares, y la subsiguiente implantación del comedor escolar -más defendible- y del transporte escolar -no tanto-, con el argumento de mejorar la «calidad» $(i)$ de la enseñanza por la graduación y presencia de «especialistas», sobre todo en el tercer ciclo de la EGB, amén de otras «virtudes» formativas en la convivencia y la rentabilidad de recursos. Sea como fuere, contribuíamos así a la despoblación y merma de los núcleos rurales que, al perder la escuela, languidecían lentamente. Aparte de los peligros evidentes del transporte escolar por carreteras no siempre en las mejores condiciones y, en 
generalizó un sistema flexible de espacios, para convertir las aulas en dependencias compartidas por varios grupos-clase, para alternar intervenciones en "gran grupo», «grupos medianos» $\mathrm{y}$ "pequeños grupos» $\mathrm{O}$ trabajo «en equipo», además de la opción del trabajo «individual» ${ }^{26} \mathrm{~A}$ la par que ampliar los existentes, pues en la progresiva -por cursos académicos o años escolares- aplicación de la LGE, todo el alumnado del nuevo $4 .^{\circ}$ de EGB, que antes se bifurcaba con el paso de un contingente importante a los centros de secundaria, para iniciar el bachillerato, permanecería ahora en el nuevo sistema -con el 5. ${ }^{\circ}$ de EGB- para continuar en la $2^{\mathrm{a}}$ etapa -cursos $6 .^{\circ}, 7 .^{\circ}$ y $8 .^{\circ}-$-, llegando, pues, todos a los 14 años, lo que obligó a «reciclar» a los maestros -especialmente en contenidospara garantizar la enseñanza a estos últimos. Asimismo hubo que dar opción a los profesores de secundaria para impartir aquellos mismos contenidos, bien que con alguna preparación -a mi juicio del todo insuficiente ni seriamente planteada- de carácter didáctico y psicopedagógico, ${ }^{27}$ con tal de asumir el proyecto de la nueva EGB, obligatoria y globalizada, en el inicio, y moderadamente diversificada al final.

Así las cosas, para la apertura de curso 1971-72 se pensó en que la conferencia inaugural la impartiera el Dr. Siguán Soler, ${ }^{28}$ catedrático de Psicología en Barcelona, que lo hizo solemnemente, en la Casa de Cultura de Girona, en presencia de autoridades, docentes y un público interesado, pues el tema fue la recién «estrenada» LGE. Por mi parte, he de confesar que no compartí todas las afirmaciones de mi admirado

cualquier caso, generador de fatiga acumulada por los madrugones y largos trayectos. Al respecto, mi admirado colega y tutor Dn. Lluís María había defendido las concentraciones escolares en una colaboración, publicada en Bordón, a buen seguro documentada y bien fundamentada pedagógicamente, como para merecer el placet del director de la revista, Dn. Víctor García Hoz.

26 Era la oportunidad de poner en práctica, la doctrina de la educación «personalizada» de García Hoz que defendía las iniciativas -complementarias- de la individualización y la socialización, en diversos grados, de la enseñanza.

27 Carencia que, a mi juicio, se ha hecho endémica, por más que -o más bien «por culpa de»- un pretendido máster universitario, en secundaria, intenta paliar aquella deficiencia estructural. Otros hubiéramos visto con mejores ojos un sistema de optativas de carácter psicopedagógico, didáctico-organizacional y legal, ofertable a todas las carreras, desde el 2..$^{\circ}$ año de estudios e impartido por las facultades de Ciencias de la Educación, con un proyecto de final de estudios/grado/tesina etc., orientado a la docencia, y las subsiguientes prácticas.

28 Al que yo mantenía un profundo reconocimiento, en tanto que lo tuve de profesor, en la asignatura de Psicología General de los cursos comunes de Filosofía y Letras, y como pionero de la Psicología en España, junto con Pinillos, Illueca Valero o Secadas. 
Dn. Miguel, pero no me atreví a contradecirle, por no crear malestar entre los asistentes y autoridades. ${ }^{29}$

Un episodio relevante, en el decurso de las celebraciones en torno a la divulgación de la LGE, fue la semana pedagógica que se organizó y en la que la Inspección Provincial tuvo un papel destacado, ya que la principal invitada fue la Dra. Galino, ${ }^{30}$ que había tenido de alumna, y a la que le dirigió la tesis después, a la compañera Anna M. ${ }^{a}$ Oriol. ${ }^{31}$ Así llegó el día señalado para su intervención y fuimos a recibirla al aeropuerto de Girona, las autoridades, presididas por el gobernador civil, y los inspectores: su condición de Directora General de Ordenación Académica -creo recordar- y persona de confianza del propio ministro Villar Palasí, concitó una gran expectativa. El caso es que, en algún momento del recibimiento, me encontré justo al lado de $\mathrm{D}^{\mathrm{a}} \mathrm{M}$. ${ }^{\mathrm{a}}$ Ángeles y, por seguir la conversación que se mantenía con ella, se me ocurrió sincerarme: «Estamos preocupados, Dra Galino, por cómo ayudar a los centros a renovar -en la línea de la LGE- sus actuaciones, programas y metodologías, quienes hemos recibido una formación según los parámetros convencionales». Me contestó al punto algo así como que «Eso pasa siempre...». Y seguía: "además todo no es tan nuevo...». «Lo que hace falta es ilusión y deseos de seguir aprendiendo...». "Y vocación, claro...». "Y de eso tenéis, ¿no?».

\footnotetext{
29 Efectivamente, el Dr. Siguán se situó más como quien temía la «avalancha» de todo -o casi- el colectivo de alumnos de secundaria que, según la común -y errada- percepción, la «evaluación continua» era un "chollo», que dejaría pasar a todo el mundo, que como autoridad en Psicología, en donde hubiera podido explayarse, largo y tendido, en diseñar propuestas de intervención psicodidácticas para el profesorado y de autoaprendizaje o compartido para el alumnado y represtigiar el sistema de evaluación, riguroso, serio y a la vez humanizado, atendida la diversidad. Pero no: se centró en analizar el papel de «cuello de botella» (sic) que, en la práctica y en lo sucesivo, tendría que jugar la universidad que, hasta entonces, lo realizaban los institutos en secundaria.
}

30 Treinta años más tarde, en el curso 2000/01, tuvimos el gozo de contar, nuevamente, con $\mathrm{D}^{\mathrm{a}} \mathrm{M}$. ${ }^{\mathrm{a}}$ Ángeles -doctora honoris causa ya, de la Universitat Jaume I-, en su participación de los cien años de existencia de la Escuela Normal de Castelló, con su intervención «Las sociedades pluriculturales requieren una educación intercultural». Vid: AA. VV., Centenari dels estudis de Magisteri a Castelló de la Plana (Castelló de la Plana: UJI, 2004), 99-115. Justamente y en la misma edición me cupo el honor de colaborar con la glosa de la vida académica de los años sesenta, como exalumno de aquella Escuela: «Fer de mestre», 51-77.

31 Justamente aquel mutuo conocimiento y confianza, derivó en alguna anécdota como la de la llamada que hizo Anna M. ${ }^{a}$ al Ministerio, al teléfono de la Dirección General preguntando por la Dra. Galino, para concretar su intervención en la semana aludida y recibió por respuesta un «Soy yo, ¿quien la llama?». Y cuando la compañera Oriol se presentó, y se extrañó de que la propia directora general descolgara el teléfono, lo consideró del todo natural. Aquella buena relación aseguró su presencia en Girona y una agradable estancia de $\mathrm{D}^{\mathrm{a}} \mathrm{M}^{\mathrm{a}}{ }^{\mathrm{a}}$ Ángeles, cuando de seguro que otros temas de más calado le ocupaban por aquellas fechas en Madrid. 
Más o menos, con miradas cómplices de alguno de los acompañantes que entreveían un reproche: «Eso te pasa por preguntar». El caso es que en su intervención, con una sala atestada de docentes y un público diverso, se ganó al auditorio y lo predispuso muy favorablemente a afrontar los nuevos tiempos de «reforma», cosa que nos complació especialmente a los inspectores.

Obviamente aunque los funcionarios de la Inspección aparecíamos como «protagonistas» de la difusión y puesta en práctica de la LGE, necesitábamos como el que más, recibir información y formación al respecto. ${ }^{32} \mathrm{~A}$ los de la promoción del 70 -la mía- ya nos habían convocado a unas sesiones en la Complutense -en los locales de la ciudad universitaria, dónde realizamos buena parte de las pruebas de las oposicionespara ser «aleccionados» por las figuras del momento, encabezadas por Dn. Víctor García Hoz. ${ }^{33}$

De otro lado, unos meses más tarde, la propia Dirección General de Ordenación Educativa organizó, en la Universitat Politècnica de València, unas sesiones sobre evaluación, a las que fuimos invitados inspectores de toda España, más o menos «portavoces» de la LGE en nuestras respectivas provincias, que coordinó la persona de confianza de la Dra. Galino y subdirector general, el profesor Ricardo Marín, con diversas intervenciones de expertos españoles y foráneos y visitas puntuales. ${ }^{34}$

Uno de los aspectos que con ocasión de la LGE -aunque no directamente derivado de su aplicación- que cobró protagonismo fue la moderada especialización -en forma de «ponencias»- a que nos vimos abocados los inspectores «generalistas». En mi caso, además de la atención a la progresiva implantación de los servicios de transporte escolar y comedores,

\footnotetext{
32 Siempre me ha parecido exigible por imprescindible, la formación continua de los funcionarios de la Inspección: «estar al día» y seguir profundizando y documentándose en temas socioeducativos y de técnicas de intervención, resulta consubstancial a la función inspectora educativa. Al respecto, recuerdo el proyecto de sesiones formativas que promovió Gonzalo Gómez Dacal -de mi misma promoción, por cierto- recién nombrado Inspector General del MEC.

33 Que para los que no habíamos estudiado en Madrid y no lo conocíamos personalmente era un semidiós, por el que sentíamos «devoción» y que resultó muy ameno e interesante, a la par que próximo y amable.

34 Como la de la, entonces, Universidad Laboral de Cheste -Centro de Orientación de Universidades Laborales era su nombre y cometido-, que nos dio la oportunidad de conocer una tal experiencia y a su Rector, el emblemático y admirado -como estudiantes en tiempos- Dr. Illueca Valero.
} 
a nivel provincial, fue la coordinación de la educación especial y el impulso y seguimiento de las intervenciones del equipo -tres personas- de los recientemente creados SOEVs -Servicio de Orientación Escolar y Vocacional. Respecto a la educación especial era el comienzo de la detección del alumnado con alguna singularidad y la decisión de atenderlo en el centro ordinario -con algún refuerzo añadido- o en aulas específicas -cuya creación se impulsó- o en centros también específicos y en las poblaciones más importantes. Hay que decir que tuve la suerte de contar con un colaborador muy dedicado y eficiente, ${ }^{35}$ a quien debo en gran medida las intervenciones de coordinación y mejora del tratamiento de un tema tan delicado, y aún polémico, como la educación especial -ahora «atención a la diversidad». Al lado de aquellas intervenciones tenían una presencia social importante las recientemente creadas asociaciones de padres y madres de Educación Especial, que se convirtieron en interlocutores indispensables para la programación de actuaciones al respecto y la intermediación con las autoridades.

En cuanto al equipo de psicopedagogos -tres- que tuve la oportunidad de coordinar en el SOEV, también a nivel provincial, se llegó a realizar una labor encomiable e inédita hasta entonces en los centros, de orientación y refuerzo de la dimensión y tratamiento psicológico general del alumnado y puntual de los casos y situaciones específicas, en parte relacionadas con el ámbito de la educación especial. Era el embrión de lo que con el tiempo derivó en la ampliación de efectivos y constitución de equipos de comarca -para la Enseñanza Primaria- y la creación e incorporación de las plazas de profesor de Psicología y Orientación, de los Institutos de Educación Secundaria.

Así pues, en aquellos primeros tiempos de mi ejercicio profesional en la Inspección Educativa, coincidentes y en buena media favorecidos por la aplicación de la LGE, tuve -tuvimos- ocasión de «transitar» por situaciones, episodios y oportunidades irrepetibles y muy valiosos para «equiparnos» de habilidades con las que llevar a buen término la función inspectora en lo sucesivo y, en mi caso al menos, hasta el final de mi

\footnotetext{
35 Efectivamente, el director del Centro «Mater Dei» de la ciudad de Girona, Agustí Quintana -de familia de médicos y viudo de la profesora de Geografía e Historia de la Normal-, tenía una formación universitaria especializada y larga experiencia y práctica docente, además de un trato amable y próximo. Obviamente, el reconocimiento público y agradecido, por mi parte, de su valía y dedicación consolidó nuestra relación que redundó en beneficio de los centros, el profesorado, las familias y los mismos alumnos de toda la provincia -de las unidades de Educación Especial y el tratamiento de la diversidad en las ordinarias.
} 
actividad profesional. Unas relacionales y comunicativas -con el alumnado, los docentes y directivos, los técnicos, las autoridades, las familias.... ${ }^{36}$ Otras de gestión -siempre amenazada la función inspectora de excesiva burocratización. ${ }^{37}$ Aprendimos «nuevos» contenidos y metodo$\operatorname{logías}^{38}$-con el peligro de confundir fines y medios y caer en el «fichismo» didáctico- con la presentación de materiales que las editoriales se apresuraron a «facilitar» a los centros, desvirtuando la esencia de las intervenciones y las iniciativas de alumnos y maestros.

Pero habrá que ir cerrando el «caos» de vivencias ${ }^{39}$-sin referencias ni bibliografía, fuera de los apuntes que aparecen en alguna nota a pie de página- que la generosidad de la revista me ha permitido retrotraer de las memorias de un joven -entonces-inspector escolar, para afirmar con tanta rotundidad como atrevimiento -y quisiera equivocarme(!)- que más de un postulado de la LGE ${ }^{40}$ está por alcanzar, como la estructura

36 Una de las «enseñanzas» que uno aprendió entonces fue la del «tacto» en relacionarse con el personal docente, tratando de mostrarse «sistemáticamente» discreto y afable en el trato. Hasta el punto de que, con ocasión de formar parte de un tribunal de oposiciones de maestros, la compañera vocal -profesora Boix-, catedrática de Pedagogía de la Escuela Normal, respecto a la relación que manteníamos los componentes, me inquirió: «!Qué respeto te tienen los maestros -del tribunal-, Marc!» No lo pensé dos veces: «!El mismo que yo a ellos, Mercé!». La misma deferencia intenté imprimir en el trato con las autoridades locales, ignorando a propósito su orientación ideológica y partidista, para ofrecer -indiscriminadamente- colaboración y reconocimiento de parte de la Inspección. O las familias, dispensando determinadas «intromisiones», en opinión de algunos docentes, para favorecer su implicación consecuente en la formación de sus hijos. Y hasta el trabajo coordinado y corresponsable con los compañeros y compañeras de plantilla -entonces y siempre.

37 El paso -traspaso- de responsabilidad -y la subsiguiente autoridad y prestigio de la Inspección -y los inspectores- a la recientemente creada Delegación Provincial del MEC -y a la figura del «delegado»-, fue mal entendida por nuestra parte. Como la que considerábamos como «competencia»-o directamente «usurpación» de funciones-, por parte de los Institutos de Ciencias de la Educación, en la intervención pedagógica y de orientación del profesorado, ejercida hasta el momento por la propia Inspección.

$38 \mathrm{La}$ «palma» se la llevaban las matemáticas «modernas» que llegaron a «desbancar» a las convencionales, así como la lengua que incorporó también nuevas terminologías. Por su parte, las intervenciones orales y expositivas -magistrales- fueron denostadas y substituidas por la exclusiva -o casiiniciativa del alumnado -y de cada alumno- y la pasiva -o casi- mirada del maestro, por no «desvirtuar» aquella iniciativa.

39 Que Mayordomo («El legado de las voces y los escritos», 5) citando a Marc Bloch abona «tener en cuenta cómo esos testimonios personales resultan irreemplazables», bien que refiriéndose a Todorov defiende que resulta «esencial en aquella mencionada operación historiográfica que inexcusablemente» ha de ser «activa, argumentada, y crítica» («El legado de las voces y los escritos», 6). Tarea, en todo caso, encomendada a los historiadores, a los que se les supone la objetividad y rigor, ausente -supuestamente también- en los «relatos» personales.

40 El mismo Profesor de Puelles (Política y Educación en la España contemporánea) lo autentifica: «La concepción global de la reforma era moderna y, en muchos de sus aspectos, sigue siéndolo, 
de las enseñanzas -EGB/ESO/ESNO. ${ }^{41} \mathrm{O}$ planteamientos como el de la evaluación continua. ${ }^{42}$ Pero algo habrá que dejar a las futuras generaciones de docentes, técnicos de la educación y políticos del futuro. Y a los propios protagonistas del sistema educativo -alumnado-43 que esperemos no se vean en la necesidad de promover una «revuelta», para que algo cambie -a mejor si puede ser- en educación. ¡Ojalá!

\section{REFERENCIAS}

Adell Cueva, Marc Antoni. «Fer de mestre». En AA. VV., Centenari dels estudis de Magisteri a Castelló de la Plana, 51-77. Castelló de la Plana: UJI, 2004.

De Puelles, Manuel. Política y Educación en la España Contemporánea. Madrid: UNED, 2004.

Galino, María Ángeles. «Las sociedades pluriculturales requieren una educación intercultural». En AA. VV., Centenari dels estudis de Magisteri a Castelló de la Plana, 99-115. Castelló de la Plana: UJI, 2004.

Mayordomo, Alejandro. «El legado de las voces y los escritos: su valor como testimonio de la cultura escolar». Revista Iberoamericana do Patrimônio Educativo 6 (2020): 1-31.

hasta el punto de que algunos de los elementos de la reforma de 1990 no son sino un nuevo intento de implantar, y a veces de profundizar, en aspectos propios de esta ley».

41 Aún ahora se me hace difícil de entender la decisión de la administración educativa del momento de «cercenar» los cursos de la $2^{\mathrm{a}}$ etapa de la EGB $-7 .^{\circ}$ y $8 .^{\circ}-$, no tanto para convertirlos en $1 .^{\circ}$ y $2 .^{\circ}$ de la Educación Secundaria Obligatoria (ESO), cuanto para «ubicarlos» físicamente, en otro espacio y ámbito distinto y demasiadas veces distante: los Institutos de Educación Secundaria. Quien tenga cerca de casa un centro público de Secundaria -y los «profes» avisados lo pueden ver cada día en espacios comunes y patios-, lo mal que «casan», criaturas de 12 años -de $6 .^{\circ}$ de Primaria a $1 .^{\circ}$ de ESO-, abrumados de libros en las mochilas de la espalda, con adolescentes granados haciendo bromas entre ellos o con las alumnas, demasiadas veces con «algo» mascullando o «inhalando». Los responsables de la enseñanza privada se comportaron bastante más inteligentemente, manteniendo aquellas etapas en un mismo «recinto» y ámbito de intervención con profesorado compartido, bien que en espacios más o menos diferenciados, pero conectados. Y no solo porque las familias -algunas no necesariamente «confesionales»- ven así garantizadas la secuencia y continuidad de las enseñanzas impartidas a sus hijos, lo que supone algo más que evitación de molestias añadidas con el cambio de centro, como ocurre, obligadamente, en la enseñanza pública.

42 «[...] el proceso de evaluación continua que, lamentablemente, sería pronto desvirtuado y falsificado por la práctica educativa». Así de contundente es el propio Manuel de Puelles, Política y Educación,

43 A los que autor ha dedicado atención preferente, en sus investigaciones y estudios. Vid.: http:// noublocdemarc.blogspot.com/p/blog-page_21.htm. 